\title{
The Cold Land Processes Experiment (CLPX-1): analysis and modelling of LSOS data (IOP3 period)
}

\author{
Marco Tedesco ${ }^{1}$, Edward J. Kim ${ }^{2}$, Don Cline ${ }^{3}$, Tobias Graf ${ }^{4}$, Toshio Koike ${ }^{4}$, Janet Hardy ${ }^{5}$, Richard Armstrong ${ }^{6}$ and \\ Mary Brodzik ${ }^{6}$ \\ 1 NASA Cold Land Processes Working Group, NASA Goddard Space Flight Center, Microwave Sensors Branch \\ 2 Laboratory for Hydrospheric Processes, NASA Goddard Space Flight Center \\ 3 National Operational Hydrologic Remote Sensing Center, National Weather Service, NOAA \\ 4 University of Tokyo \\ 5 Cold Regions Research and Engineering Lab., USACE \\ 6 National Snow and Ice Data Center, Univ. of Colorado \\ mail to: mtedesco@umbc.edu
}

\begin{abstract}
Microwave brightness temperatures at 18.7, 36.5, and $89 \mathrm{GHz}$ collected at the Local-Scale Observation Site (LSOS) of the NASA Cold-Land Processes Field Experiment in February, 2003 (third Intensive Observation Period) were simulated using a Dense Media Radiative Transfer model (DMRT), based on the Quasi Crystalline Approximation with Coherent Potential (QCACP). Inputs to the model were averaged from LSOS snow pit measurements, although different averages were used for the lower frequencies vs. the highest one, due to the different penetration depths and to the stratigraphy of the snowpack. Mean snow particle radius was computed as a best-fit parameter. Results show that the model was able to reproduce satisfactorily brightness temperatures measured by the University of Tokyo's Ground Based Microwave Radiometer system (GBMR-7). The values of the best-fit snow particle radii were found to fall within the range of values obtained by averaging the field-measured mean particle sizes for the three classes of Small, Medium and Large grain sizes measured at the LSOS site.
\end{abstract}

Keywords- Snow, Microwave remote sensing, DMRT

\section{INTRODUCTION}

Snow is a critical element of the Earth's hydrologic cycle. The northern hemisphere land surface is covered by snow in midwinter for more than $60 \%$, and over $30 \%$ of Earth's total land surface has seasonal snow [1]. In many mountainous regions of the Earth, snowfall represents the majority of precipitation and snowmelt is responsible for most of the total annual streamflow. Moreover, snow covers are fundamental in the Earth's energy cycle, and through their effects on land surface albedo, the net radiation balance, and boundary layer stability, have profound affects on weather patterns over large areas.

The Cold Land Processes Field Experiment-1 (CLPX-1) was designed to advance the understanding of the terrestrial cryosphere and cold areas of the Earth's land. The CLPX-1 aimed at developing a more complete understanding of fluxes, storage, and transformations of water and energy in cold land areas, being this a critical focus of the NASA Earth Science Enterprise Research Strategy, the NASA Global Water and
Energy Cycle (GWEC) Initiative, the Global Energy and Water Cycle Experiment (GEWEX), and the GEWEX Americas Prediction Project (GAPP). Intensive ground, airborne, and spaceborne observations were collected in Colorado, central Rocky Mountain of the western United States, during February and late March of both 2002 and 2003. In total, four week-long Intensive Observation Periods (IOPs) were conducted in order to observe dry (IOP1 and IOP3, February 2002 nd 2003) and wet (IOP2 and IOP4, March 2002 and 2003) snow conditions. A set of nested study areas, ranging from 1 ha to $160,000 \mathrm{~km}^{2}$, was considered to permit a detailed examination of cold land processes, modeling, and measurement over a wide range of physiographic conditions and spatial scales.

To our aim, brightness temperatures at $18.7,36.5$, and 89 $\mathrm{GHz}$ acquired by means of the University of Tokyo's Ground Based Microwave Radiometer-7 (GBMR-7)during the third Intensive Observation Period (IOP3, dry snow), February 19 25, 2003 at the Local Scale Observation Site (LSOS) test site were analyzed in conjunction with nivological measurements.

The LSOS test site data were ideal for our aim consisting of relating snow pit measurements and radiometric observations respectively with inputs and outputs of an electromagnetic model based on Dense Media Theory under the QuasiCrystalline Approximation (DMRT, QCA-CP) ([2]-[5]). Indeed, at this test site, temporal and spatial intensive measurements of snow properties were performed several times during each single day in conjunction with the collection of radiometric data. The mean particle radius in the electromagnetic model was used as a best-fit parameter. It was computed by minimizing the mean square error (MSE) between measured and simulated brightness temperatures. All other parameters in the DMRT model were derived from snow field measurements collected close in time and space to radiometric acquisitions. As the electromagnetic model was a single layer model, snow parameters measured along the vertical profile were averaged and used as inputs to the model Different values of thickness were used to average snow parameters at different frequencies because of the different penetration depths. Simulated and measured radiometric 
quantities were, then, compared as well as values of mean grain diameters obtained by the model with average ice grains size.

\section{THE TEST SITE, SNOW PIT DATA AND RADIOMETRIC SYSTEM}

The test site consisted of a small (0.8-ha) clearing surrounded by trees and located within the CLPX Fraser Intensive Study Area (ISA), near the Fraser Experimental Forest Headquarters Facility, Colorado (105'54'40' W, 39 $50^{\prime} 49^{\prime}$ 'N, $2780 \mathrm{~m}$ a.s.1.) (Figure 1. ).

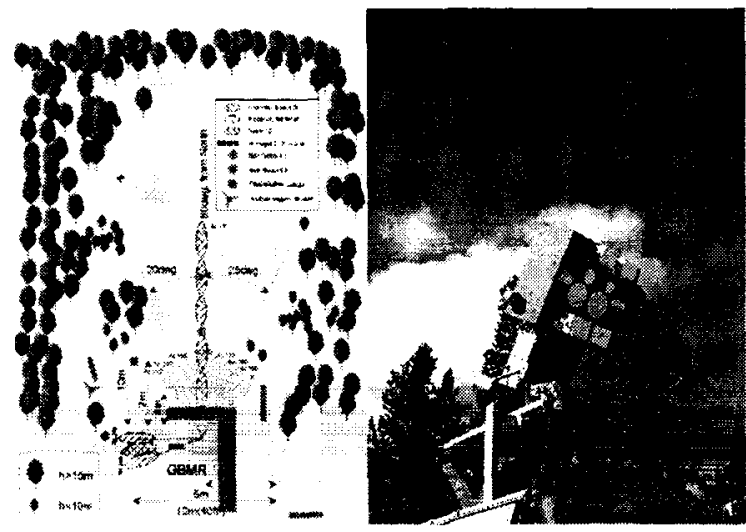

Figure 1. Sketch of the LSOS test site (left) and picture of the antennas and positioning system of the GBMR-7 operating at LSOS test site (right).

Measurements of several snow properties were collected daily at different locations in the small clearing at the LSOS. Snow properties such as snow depth, density, temperature, grain size distribution, and stratigraphy. were measured from the vertical wall of each pit using a well-established protocol Snow depth was measured to the nearest $0.01-\mathrm{m}$ using a collapsible, graduated snow probe. Snow density was measured at $10-\mathrm{cm}$ intervals using a $1000-\mathrm{cm}^{3}$ snow density cutter and electronic scale. Snow temperature was measured at $10-\mathrm{cm}$ intervals using a calibrated dial-stem thermometer. Three measures of grain size distribution, respectively called Small, Medium and Large sizes, were made in each layer using a microscope with 8 $\times 30$ optics. Snow temperature recorded at different heights of the snowpack never exceeded $0^{\circ} \mathrm{C}$ during the whole IOP3 period, assuring the conditions of dry snow. As an example, Figure 2. shows the temporal trend of densities averaged along different thickness and snow depth. It is possible to observe that the average density along the total depth is weakly influenced by snow precipitations. The countrary happens for those values of density averaged along the first centimeters.

Microwave data were acquired by means of the ground based passive microwave radiometer GBMR-7 (University of Tokyo), which was developed for environmental research and designed for extreme outdoor conditions [6]. Brightness temperatures at $18.7,36.5$ and $89.0 \mathrm{GHz}$ (both vertical and horizontal polarizations) were measured [7]. Incidence angle scans were performed with observation angle ranging between 30 and 60 degrees and with a fixed azimuth angle of $180^{\circ}$. Azimuth scans were also performed with azimuth angle ranging between $170^{\circ}$ and $190^{\circ}$ with a fixed incidence angle of $55^{\circ}$. All critical parts of the receivers were encapsulated in a thermally-stabilized radiometer box which was mounted on an accurate positioning system (Figure 1. ). The GBMR-7 used a combination of the cold/hot and Dicke switching techniques to calibrate the radiometers. Another feature, suiting the system for environmental research, was the use of a second internal calibration standard. The complete system was computercontrolled.

\section{INPUT PARAMETERS AND MODELLING RADIOMETRIC DATA USING THE DMRT MODEL}

In the electromagnetic model, snow-pack is modeled as a slab of densely distributed spherical particles having radius $a$ and permittivity $\varepsilon_{i}$, embedded in a background medium (assumed to be non-absorptive, i.e. air) of permittivity $\varepsilon_{b}$ and lying above a ground with permittivity $\varepsilon_{g}$. The effects of roughness at the air-snow and snow-ground interfaces were disregarded but the consequences were preliminary analyzed. Obtained results show that, if all parameters are fixed with the exception of mean particle size, the highest error introduced on the mean particle size is about $0.03 \mathrm{~mm}$. As stated, we extrapolated inputs to the electromagnetic model by averaging the measurements carried out along the snow-pack vertical profile. The penetration depth was estimated using the DMRT model and literature data (i.e. [8],[9]). It resulted that at low frequencies ( 18.7 and $36.5 \mathrm{GHz}$ ), the penetration depth is of the order of one meter where at $89 \mathrm{GHz}$ it is of the order of few centimeters. It happens, therefore, that at 18.7 and $36.5 \mathrm{GHz}$, the penetration depth is deeper than, or comparable to, the snow-pack depth and, hence, the total height of the snow-pack was used as averaging thickness. At $89 \mathrm{GHz}$, only the first 10 centimeters were used to calculate the averaged snow parameters because of the small penetration depth.

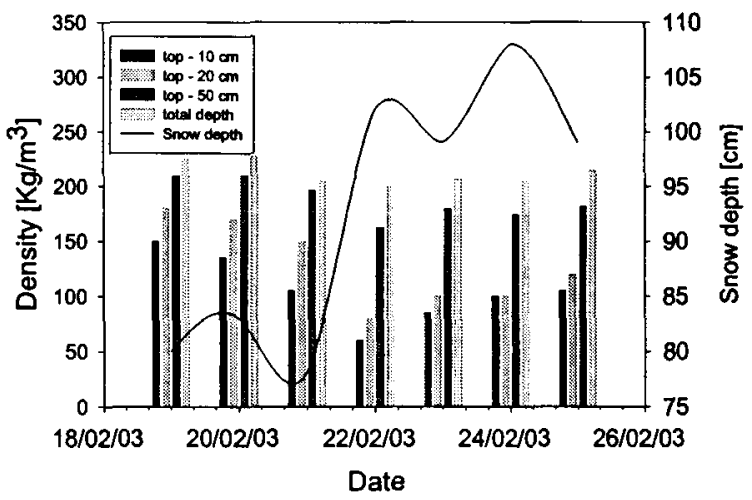

Figure 2. Temporal trend of densities averaged along different thickness and snow depth

As stated in the Introduction, to model radiometric data all model inputs were kept fixed with the exception of mean particle size, which was used to as a best fit parameter to minimize the Mean Square Error (MSE) between simulated and measured brightness temperatures. Ice permittivity was computed according to the formula proposed by Hufford [10]. As an example, Figure 3. compares simulated and measured brightness temperatures at 18.7 (a), 36.5 (b) and $89 \mathrm{GHz}(\mathrm{c})$, as 
a function of the observation angle on February 19, 2003. The values of input parameters in this case were: $T_{\text {snow }}=269.5 \mathrm{~K}$, $\mathrm{T}_{\text {ground }}=272.5 \mathrm{~K}$, fractional volumes $f_{\text {low }}=0.3$ at low frequencies and $f_{\text {high }}=0.21$ at high frequencies, snow depth $d=$ $0.8 \mathrm{~m}, \varepsilon_{\text {ground }}=4.5+\mathrm{i}^{*} 0.1$, and the value of mean particle radii derived from the minimization of MSE were $a=0.56 \mathrm{~mm}$ at 18.7 and $36.5 \mathrm{GHz}$ and $a=0.27 \mathrm{~mm}$ at $89 \mathrm{GHz}$.

\section{RESULTS AND DISCUSSION}

Obtained results for the whole IOP3 period indicated that the DMRT model was able to simulate measured brightness temperatures as a function of the incidence angle with low error when using grain size as optimization parameter. It is important to point out again that all remaining parameters used for modeling were based on quantities measured in the field. The relative error between simulated and measured brightness temperatures was calculated at all frequencies ,polarizations and angles ranging between $30^{\circ}$ and $60^{\circ}$, with a $5^{\circ}$ step. In general, highest errors were observed, at high observation angles, with the error decreasing as the incidence angle decreases. For observation angles lower than $40^{\circ}$ the percentage error did not exceed $2 \%$, with the vertical polarization showing an error higher than or comparable to the horizontal one. With observation angles ranging between $40^{\circ}$ and $50^{\circ}$, the error at $18.7 \mathrm{GHz}$ and $36.5 \mathrm{GHz}$ decreased for horizontal polarization and increased in the case of vertical polarization. The error increased again as the observation angle exceeds $50^{\circ}$, reaching a maximum for observation angles around $60^{\circ}$. To justify the behavior of the error as a function of the observation angle, we must consider that the model is a single layer model and that ice particles are approximated by spheres. In general, ice crystals are of irregular shape but, in the model, they are approximated by spheres with radius $a$, the latter being the mean value of a particle size distribution [11]. In the case of significant stratigraphy, polarization effects are induced by discontinuities among different layers and become stronger as the observation angle increases and in our case, a melt-freeze basal ice layer (discontinuous and variable depth) was observed at the bottom of the snow/pack. Error behaviour as a function of observation angle can be very important if we want to perform an inversion of snow parameters from measured radiometric data by inverting the model (i.e. throughout numerical techniques). Indeed, if we are considering observations at $30^{\circ}$ and $50^{\circ}$, we must consider that in the case of the lowest angle the error due only to the model is very low whereas it decreases at $50^{\circ}$. As stated, three classes of grain size were measured on the LSOS test site: Small, Medium and Large. For each class, measurements along the snow-pack vertical profile were provided and values of maximum and minimum measured grain sizes at the same depth and for the same class were averaged. In example, on February 19, the top 10 centimetres average values for Small, Medium and Large grain size were, respectively, $0.15 \mathrm{~mm}, 0.2$ $\mathrm{mm}$ and $0,35 \mathrm{~mm}$.
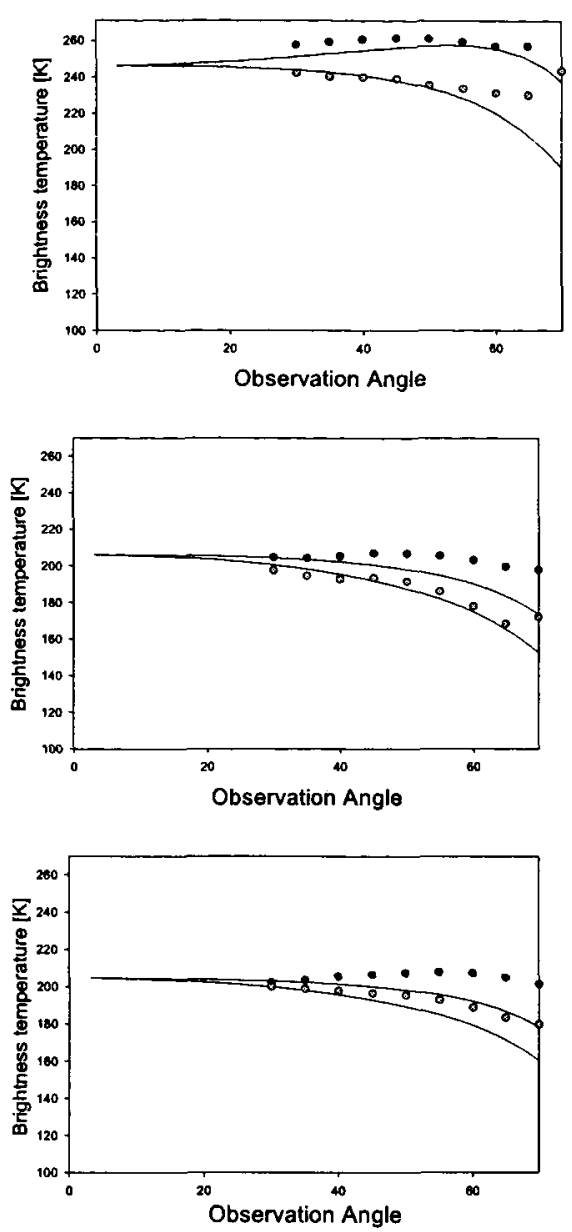

Figure 3. Simulated (lines) and measured (dots) brightness temperatures for February 19, 2003 as a function of the observation angle at 18.7, 36 and 89 $\mathrm{GHz}$

By this way, average values of the maximum and minimum radius measured at the same depth were obtained. Then, they were used to calculate the average value along the profile for the three classes of Small, Medium and Large grain sizes. Values obtained in this fashion were compared with those found by the DMRT model. Note that, as already stated, due to different penetration depths, different averaging depths were used for the highest $(89 \mathrm{GHz}$ ) and for the lower frequencies $(18.7$ and $36 \mathrm{GHz})$. It is important to note that we computed the grain radius from the model separately for each frequency and, then, noted that the values corresponding to low frequencies were very similar between them but different from that obtained at $89 \mathrm{GHz}$, which were found to be smaller than those retrieved at low frequencies. Figure 4 . show the temporal trend of the diameters computed with the DMRT model (diamonds) at 18.7 and $36.5 \mathrm{GHz}$ (up) and $89 \mathrm{GHz}$ (down) together with average values of Small, Medium and Large particle sizes It appears interesting to point out the instability of temporal trend of values of average measured grain sizes, mainly due to metamorphism. This instability is not present in the retrieved values of mean particle diameter. To explain this, it is very 
important to remind that the mean particle radius in the DMRT model represent the mean of a log-normal probability density function describing the particle sizes distribution. Indeed, values retrieved by the model are in good agreement with the mean of the distribution of snow grain sizes computed in [12] where the distribution of snow particles in artificially produced snow is computed by counting the number of particles having dimension in different ranges of values. Obtained distributions are similar to log-normal distribution (that one used in the DMRT model) and the values of mean particle size retrieved by the inversion of the DMRT model correspond, as expected, to the mean of the grain size distribution for metamorphosed snow at low frequencies and to new or metamorphosed snow in the high frequency case.
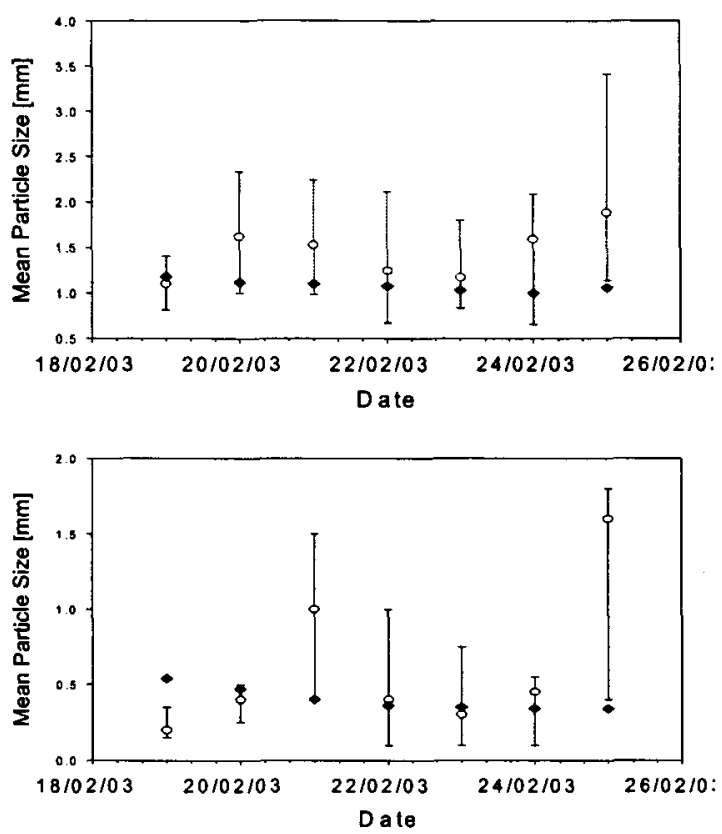

Figure 4. Diameters computed with the DMRT model (diamonds) at 18.7 and $36.5 \mathrm{GHz}$ (up) and $89 \mathrm{GHz}$ (down) together with average values of Small, Medium and Large particle sizes.

\section{CONCLUSIONS}

Analysis and modelling of the radiometric and nivological ground-based data from the LSOS test site, collected on February 19, 2003, during IOP3 period were carried out.

Microwave data, collected by the University of Tokyo's GBMR-7 radiometer at different incidence angles $\left(30^{\circ}-60^{\circ}\right)$ and frequencies $(18.7,36.5$ and $89 \mathrm{GHz})$, were compared with simulations performed using a DMRT model, based on the QCA-CP. Obtained results were very encouraging as the model was able to reproduce the measured brightness temperatures, with a minimum percentage error of about $2 \%$ for low observation angles $\left(30^{\circ}\right)$ and a maximum error lower than $7 \%$ for high incidence angles $\left(60^{\circ}\right)$. Model inputs were represented by the average values of measured snow parameters, with the exception of the mean particle radius, which was computed as a free best fitting parameter. The values of the diameter that resulted in a best fit of the experimental data were found to be in good agreement with the average values of mean particle size measured at the test site and with expected value of mean of the distribution describing particle size. The consistency of the values measured with those ones used as model inputs is an encouraging factor for the comparison between retrieved and measured snow parameters. As future work, a study considering IOP4 data (wet snow case) is under study as well as the comparison among ground based, airborne and satellite collected data to explore scaling issues related to microwave remote sensing of snow.

\section{ACKNOWLEDGMENT}

The authors wish to thank Hideyukii Fujii (University of Tokyo), JST (Japan Science and Technology Coporation) and JAXA (Japan Aerospace Exploration Agency)..

\section{REFERENCES}

[1] Robinson, D., Dewey, K., and R. Heim, Global snow cover monitoring An update, Bull. American Meteorological Society 74, 1689-1696, 1993.

[2] L. Tsang, J. A. Kong and R. T. Shin, Theory of microwave remote sensing, Wiley-Interscience, 1985

[3] Y.-Q. Jin, Electromagnetic scattering modelling for quantitative remote sensing, World Scientific Press, 1993

[4] M. Tedesco, Solution of radiative transfer equation applied to dry snow employing dense medium theory, Report Institute of Research on Electromagnetic Waves 'Carrara', Italian National Research Council, Microwave Remote Sensing Group, n. RR-TR-04.00, ISSN 1120-2823 ,April 2000

[5] M. Tedesco, Microwave remote sensing of snow, $\mathrm{PhD}$ Thesis, Institute of Applied Physics 'Carrara', Italian National Research Council, IFAC CNR, Microwave Remote Sensing Group, November 2003

[6] S. Kazama, T. Rose, and R. Zimmerman. "A Precision Autocalibrating 7ch Radiometer for Environmental Research Applications" Journal of The Remote Sensing Society of Japan, Vol. 19, No. 3, pp. 37-45, 1999

[7] T. Graf, T. Koike, H. Fujii, M. Brodzik, and R. Armstrong. 2003. "CLPX-Ground: Ground Based Passive Microwave Radiometer (GBMR-7) Data". Boulder, CO: National Snow and Ice Data Center. Digital Media.

[8] C. Maetzler, Application of SMOS over terrestrial ice and snow, $3^{\text {rd }}$ SMOS Workshop, DLR, Germany, December 2001

[9] Rott H., Sturm K. and H. Miller, Active and passive microwave signatures of Antarctic firn by means of field measurements and satellite data, Annals of Glaciology, Vol. 17, 1993, pp 337-343.

[10] G. Hufford., A model for the complex permittivity of ice at frequencies below $1 \mathrm{THz}$, Int. Journ. Of Infrared and MM Waves, Vol. 12, No. 7 , 1991

[11] G.Macelloni, S. Paloscia, P. Pampaloni and M. Tedesco, "Microwave Emission from Dry Snow: A Comparison of Experimental and Model Result," IEEE Trans. Geosci. Remote Sensing, vol. 39, n. 12, pp. 2649. 2656, December 2001.

[12] Nakamura T., Tamura R., Ohta T. and Abe O., (2001), Experimental study on the spectral reflectance of snow, http://www.issw.noaa.gov/nakamura.htm 